\title{
AN ASSESSMENT OF THE EXPECTED QUALITY OF AEOLUS LEVEL-2B WIND PRODUCTS
}

\section{Michael P. Rennie*}

${ }^{I}$ European Centre for Medium-Range Weather Forecasts (ECMWF), Reading, UK, *m.rennie@ecmwf.int

\begin{abstract}
The ADM-Aeolus L2B horizontal line-of-sight wind products are discussed, including a brief introduction to the $\mathrm{L} 2 \mathrm{~B}$ processing methods. Realistic simulation of Aeolus followed by the Ground Segment processing chain is used to assess the expected error statistics of the L2B winds. Examples of the L2B winds simulated from realistic atmospheric cases are discussed. The aim of the paper is to give potential users an impression of the expected characteristics of Aeolus mission's main product.
\end{abstract}

\section{INTRODUCTION}

ADM-Aeolus is the fifth satellite to be launched in the Living Planet Programme of the European Space Agency (ESA) [1]. The mission's objective is to provide profiles of high-quality wind observations from the surface to around $30 \mathrm{~km}$ altitude, using the Doppler wind lidar instrument ALADIN (Atmospheric LAser Doppler Instrument) in a near-polar sun-synchronous, dawn-dusk orbit. The wind information is the horizontal line-of-sight (HLOS) component, in the off-nadir direction at $35^{\circ}$ and perpendicular to the satellite's velocity. The mission is intended to have a minimum lifetime of three years and is expected to be launched in late 2017.

The European Centre for Medium-Range Weather Forecasts (ECMWF) is closely involved in the Aeolus mission because the assimilation of the wind products is expected to significantly improve our weather forecasts, if the mission requirements [3] can be met. Wind profiles are still lacking for global Numerical Weather Prediction (NWP), particularly in the upper troposphere, lower stratosphere and away from populated continents.

ECMWF and KNMI (Royal Netherlands Meteorological Institute) are contracted by ESA to develop the Aeolus Level 2B/C processing software i.e. the retrieval of HLOS wind components from the Level 1B product suitable for use in NWP data assimilation. In the past
Météo-France, German Aerospace Center (DLR) and LMD/IPSL (Laboratoire de Météorologie Dynamique) have contributed to the L2B processor [2].

Some features of the L2B products are:

- (H)LOS wind components geolocated with geometric height, latitude, longitude, azimuth angle and time

- Flexible "grouping" algorithm in terms of horizontal averaging length-scale, see Figure 1

- Each observation type (either Mie or Rayleigh) is retrieved from accumulating measurement-level spectrometer counts after classification into clear or cloudy conditions

- Rayleigh winds are corrected for the temperature and pressure sensitivity using a priori information (from NWP model forecasts) and also corrected for Mie crosstalk

- Uncertainty estimates for each wind result and quality flags are derived and provided

- Processing options are easily controlled from a settings file

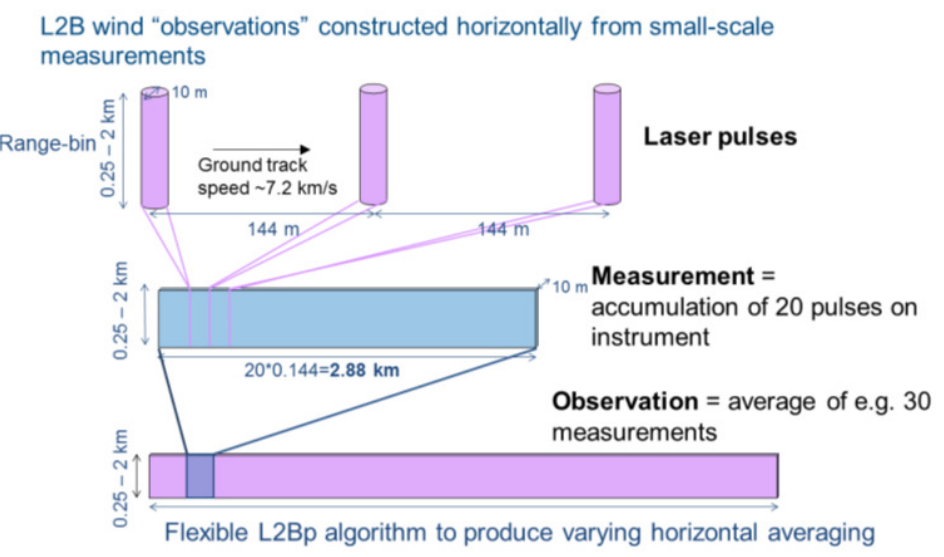

Figure 1. Illustration of how the L2B observation consists of horizontally accumulated "measurement" level data. 
The L2B processing software is portable, easy to install and is freely available for users to download from: https://software.ecmwf.int/wiki/display/AEOL/A DM-Aeolus+Level-2B+Processor+Package

ECMWF will generate the L2B products during the exploitation phase in near real-time as part of the Ground Segment and will provide the products to ESA for further distribution and archiving. ECMWF will assimilate L2B BUFR product in the global data assimilation analysis, if forecast skill is demonstrated to improve. Related to this is the Level 2C product; which provides vector winds from the ECMWF analysis after the assimilation of L2B winds.

ECMWF has over the years been involved in observation impact studies to assess the potential impact of the Aeolus data. We expect the Aeolus mission to have a positive impact on the analysis and forecast quality, in particular the improvement of winds in the tropics and in the upper troposphere [4]. This work also demonstrated that HLOS winds can provide around $70 \%$ the impact of full vector winds.

We now provide an assessment of the Aeolus L2B HLOS wind observation accuracy and precision from realistic simulations and the application of the Ground Segment processing chain.

\section{SIMULATION METHODOLOGY}

We have employed the Aeolus end-to-end simulator (E2S) and the operational chain-ofprocessors Level 0, 1A, 1B, CAL suite, and 2B) to investigate L2B wind errors in realistic atmospheric scenarios. The atmospheric inputs to the simulator, i.e. our reference "truth", are derived from ECMWF global model short-range forecasts at resolution $\mathrm{TcO} 1279$ ( 9 km horizontal grid spacing). The E2S atmospheric inputs are:

- Temperature, pressure and molecular backscatter and extinction coefficients derived from these fields

- Particulate backscatter and extinction coefficients derived from model cloud liquid and ice water content using a basic parameterization and assuming a constant lidar ratio of 20; aerosol optical properties are not considered
- HLOS wind derived from model wind components

The atmospheric scenario demonstrated here is a section of an orbit that intersects an equatorial wave with Rossby and Kelvin wave characteristics on $15^{\text {th }}$ March 2014 in the eastern Pacific. Uncertainties in the NWP wind and temperature analysis in this area associated with this wave have been shown to have contributed to a very poor forecast six day forecast over Europe. Therefore this is an interesting case to see what Aeolus observes and how it compares to real wind observations assimilated in the ECMWF operational analysis.

The optical properties and wind inputs to the tropical simulation are shown in Figure 2 and Figure 3.

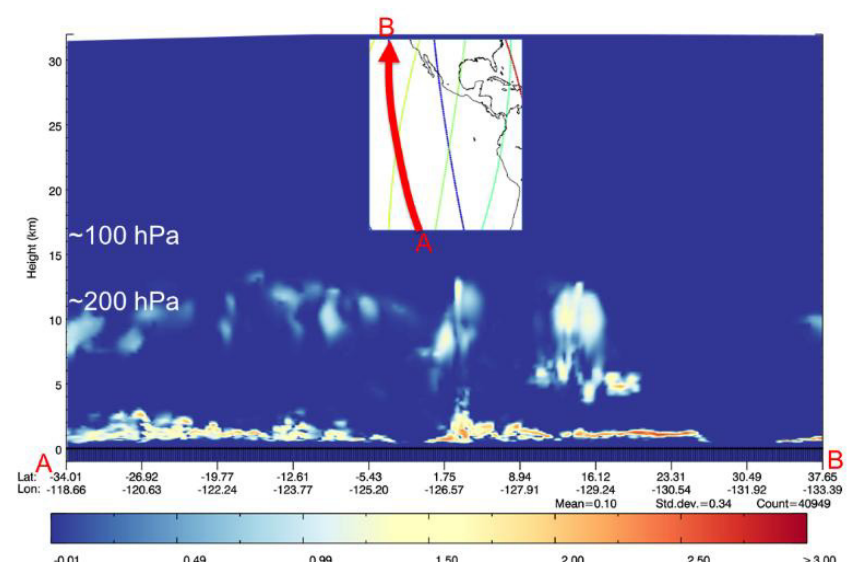

Figure 2. Cross-sectional view of end-to-end simulator input; $\log _{10}$ of the scattering ratio $\left(1+\frac{\beta_{p}}{\beta_{m}}\right)$. The map (inset) shows the orbit section (A to B).

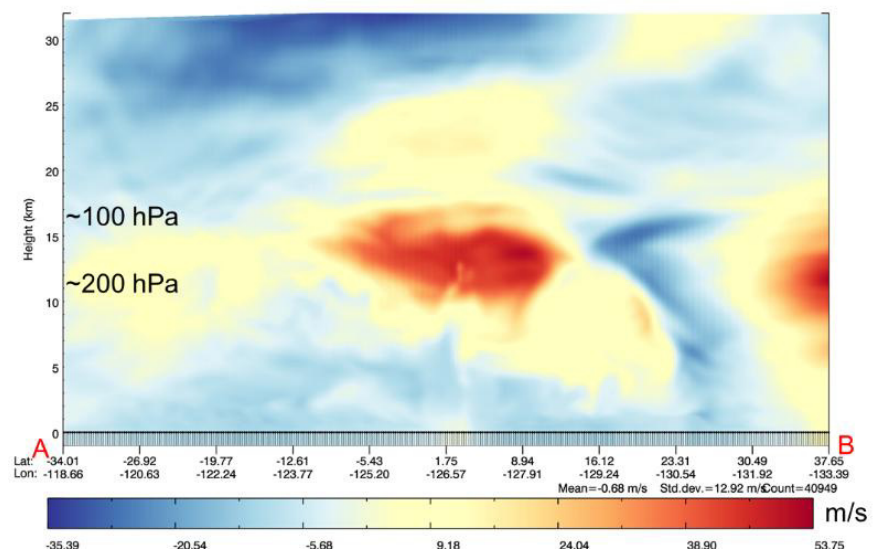

Figure 3. The end-to-end simulator input HLOS wind derived from the ECMWF model forecast i.e. truth to assess L2B winds against. Unit: $\mathrm{m} / \mathrm{s}$ 
The resulting L2B HLOS wind error statistics are examined. Interpreting these L2B error statistics comes with the caveat that the Aeolus simulator is probably not accounting for all sources of instrument noise and hence this will be an underestimate of the real Aeolus error statistics; however there are no known major deficiencies. N.B. this simulation was done with the old higher orbit $(408 \mathrm{~km})$ rather than the $320 \mathrm{~km}$ which was recently chosen.

\section{LEVEL-2B HLOS WIND RESULTS}

The resulting L2B Rayleigh-clear and Mie-cloudy HLOS wind observations are shown in Figure 4 and Figure 5 which should be compared to the "truth" HLOS wind of Figure 3 (N.B. the same scales are used for comparison).

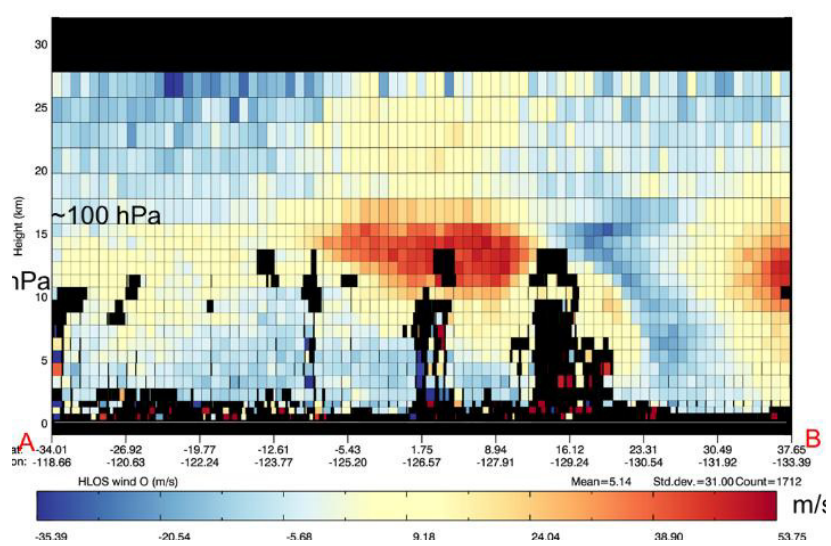

Figure 4. The resultant $L 2 B$ Rayleigh-clear HLOS wind observations, which can be compared to the "truth" HLOS winds of Figure 3.

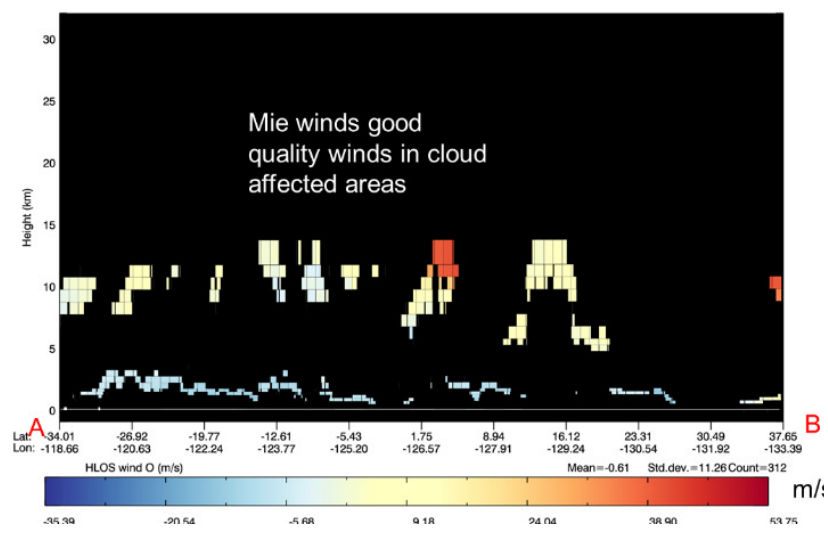

Figure 5. The resultant L2B Mie-cloudy HLOS wind observations, which can be compared to the "truth" HLOS winds of Figure 3.

Aeolus provides a blocky and somewhat noisy view of reality due to the resolution of the observations and the signal-to-noise ratio (SNR). However Aeolus Rayleigh winds are still able to capture the equatorial wave at $100-200 \mathrm{hPa}(50$ $\mathrm{m} / \mathrm{s}$ feature in the middle of Figure 3) very well. Also, notice the complementary nature of the Rayleigh and Mie results in terms of coverage.

Aeolus should substantially improve the upper troposphere and lower stratosphere wind observation coverage in the tropics compared to the status quo. The plot of real observations actively assimilated in the ECMWF analysis for this case is shown in Figure 6 (the logarithmic pressure axis and latitude axis is chosen to roughly match the lidar cross-sections for comparison). There are 3700 real observations (mostly AMVs) of u-component wind (in a $20^{\circ}$ longitude section) versus 2000 expected L2B Rayleigh-clear winds (for the Aeolus "curtain"). Only the lower portion of the equatorial wave is sampled with the current wind observing system (and there are gaps around 500-600 $\mathrm{hPa}$ ). Therefore Aeolus will provide the only direct wind information for the NWP analysis of these upper troposphere/lower stratosphere equatorial waves (apart from a few radiosondes).

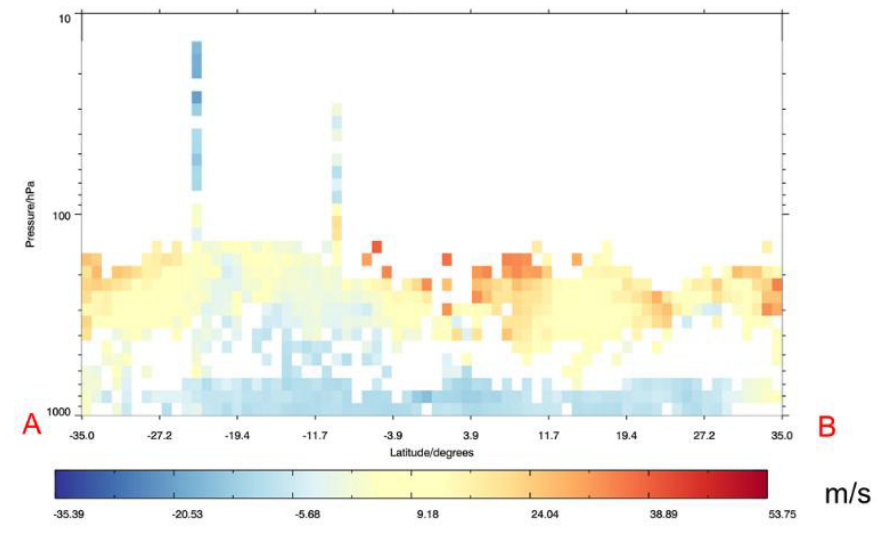

Figure 6. ECMWF actively assimilated u-component wind observations, available in the longitude range $140^{\circ} \mathrm{W}-120^{\circ} \mathrm{W}$, for the 12 hour data assimilation cycle at ECMWF on $14^{\text {th }}$ March 2014. Showing that large parts of the equatorial wave feature are not observed.

The expected Aeolus L2B wind error statistics are shown in Figure 7; from a large global sample of realistically simulated data ( 5.5 orbits); point wind values are considered as "truth". The key features are that L2B Rayleigh-clear error standard deviation is around $3-5 \mathrm{~m} / \mathrm{s}$ and Mie-cloudy around $1.5-2 \mathrm{~m} / \mathrm{s}$, with systematic errors generally 
less than $0.5 \mathrm{~m} / \mathrm{s}$. However note this used "best case" calibration files (worst case systematic errors have been estimated separately to be around $0.5 \mathrm{~m} / \mathrm{s}$ ).
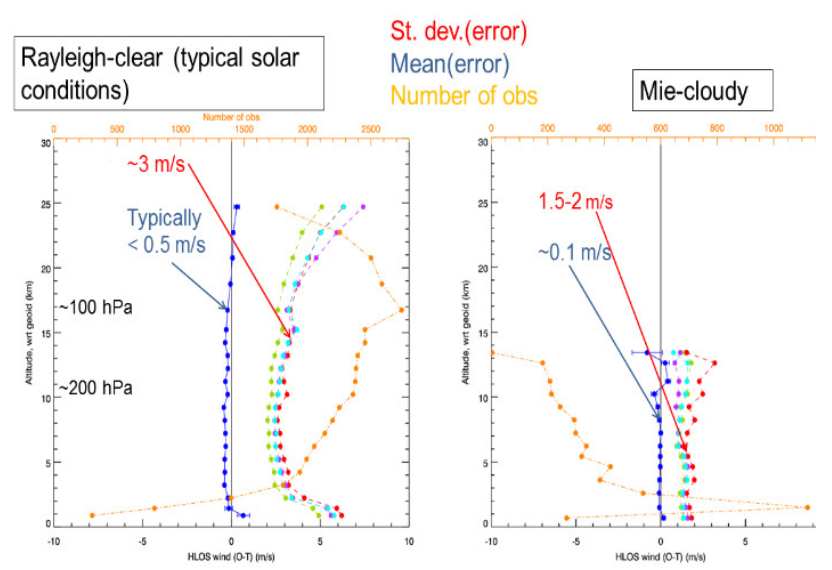

Figure 7. The level of systematic and random errors in the L2B Rayleigh-clear and Mie-cloudy HLOS winds from realistic simulations will all forms of noise "on" in the wind mode simulation. QC was applied to reject Rayleigh winds with L2B error estimates $>8 \mathrm{~m} / \mathrm{s}$ and Mie winds $>3.5 \mathrm{~m} / \mathrm{s}$; this removes outliers.

The L2B random error magnitude depends on the size of horizontal accumulation (grouping length) used in the processing; this dependence in shown in Figure 8.

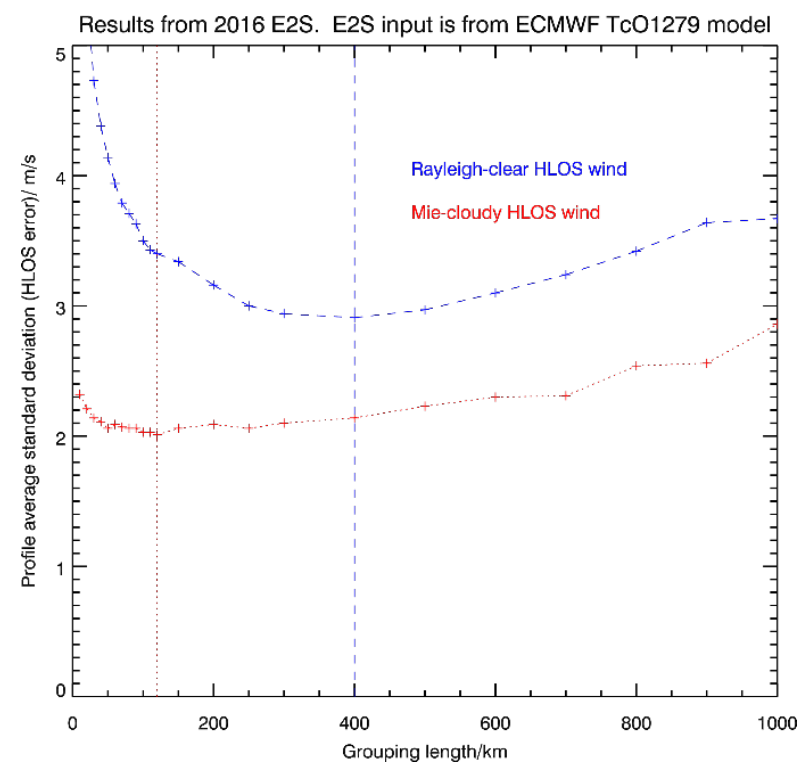

Figure 8. The dependence of the L2B HLOS wind random error (standard deviation) upon the grouping length of the L2B observations.

It is clear that much lower random errors are achievable with the Mie winds compared to the
Rayleigh for small horizontal accumulations (e.g. $20 \mathrm{~km}$ ). The Rayleigh random errors reach a minimum around $400 \mathrm{~km}$ accumulation (counteracting the Poisson noise). However not shown here is that the mean errors increase above $100 \mathrm{~km}$ averaging due to smoothing the horizontal wind shear across jet streams. In practice at ECMWF we will probably choose around $100 \mathrm{~km}$ for the Rayleigh and around 30 $\mathrm{km}$ for the Mie grouping length.

\section{CONCLUSIONS}

The simulation studies show that Aeolus L2B HLOS winds are looking promising in terms of random errors, systematic errors and sampling relative to the current observing system to achieve a significant positive impact in global NWP, particularly in the tropics where upper troposphere/lower stratosphere winds are very much lacking.

\section{ACKNOWLEDGEMENTS}

This work is funded by an ESA contract 4200018555/04/NL/MM. Thanks to Lars Isaksen (ECMWF) for advice. Thanks to KNMI (in particular Dr. Jos de Kloe) for setting up the Chain-of-Processors system that allows the endto-end testing to be run on complex atmospheric data. Thanks to the L1B and L2B teams for general support.

\section{References}

[1] European Space Agency ESA, 2008: ADMAeolus Science Report, ESA SP-1311, 121 pp.

[2] Tan, D., Andersson., E., de Kloe, J., Marseille, G.-J., Stoffelen, A., Poli, P., Denneulin, M.-L., Dabas, A., Huber, D., Reitebuch, O., Flamant, P., Le Rille, O., Nett, H., 2008: The ADMAeolus wind retrieval algorithms. Tellus 60A, 191-205.

[3] European Space Agency ESA, 2016: ADMAeolus Mission Requirements Documents, AERP-ESA-SY-001, Issue 2, 16/11/2016, 57pp.

[4] Horányi, A., Cardinali, C., Rennie, M. and Isaksen, L. (2015), The assimilation of horizontal line-of-sight wind information into the ECMWF data assimilation and forecasting system. Part I: The assessment of wind impact. Q.J.R. Meteorol. Soc., 141: 1223-1232. doi:10.1002/qj.2430 